\title{
A Parallel between \\ Brownian Bridges and Gamma Bridges ${ }^{\dagger}$
}

\author{
By \\ Michel ÉMERY* and Marc YOR** \\ I know [... ] how to construct an infinite \\ number of bridges $[\ldots]$ \\ LEONARDO, Letter to Ludovico il Moro.
}

\begin{abstract}
Some properties of the Gamma bridges (obtained by conditioning the Gamma subordinator to take a given value at a given time) are investigated; similarities with the Brownian bridges are emphasized.
\end{abstract}

\section{$\S 1 . \quad$ Introduction and Overview}

(1.1) Among the family of one-dimensional Lévy processes, the Brownian motion and the Poisson process are often compared, or discussed in close connection, for they are the only Lévy processes to share the predictable representation property, i.e.:

if $\left(B_{t} ; t \geq 0\right)$ denotes a Brownian motion and $\left\{\mathcal{B}_{t}\right\}$ its natural filtration, then every $\left\{\mathcal{B}_{t}\right\}$-martingale $\left(M_{t} ; t \geq 0\right)$ may be represented as

$$
M_{t}=c+\int_{0}^{t} m_{s} \mathrm{~d} B_{s}, \quad t \geq 0,
$$

Communicated by Y. Takahashi. Received October 6, 2003.

2000 Mathematics Subject Classification(s): 60G51, 60J75

${ }^{\dagger}$ This article is an invited contribution to a special issue of Publications of RIMS commemorating the fortieth anniversary of the founding of the Research Institute for Mathematical Sciences.

*IRMA, 7 Rue René Descartes, 67084 Strasbourg, France.

e-mail: emery@math.u-strasbg.fr

** LPMA, Université Pierre et Marie Curie, Boîte courrier 188, 4 Place Jussieu, F-75252 Paris Cedex 05, France. 
where $c \in \mathbb{R}$, and $\left(m_{s}\right)$ is a predictable process; and the same property holds for the Poisson process $\left(N_{t}\right)$ with intensity $\lambda$, the representation of the generic $\left\{\mathcal{N}_{t}\right\}$-martingale being

$$
M_{t}=c+\int_{0}^{t} m_{s} \mathrm{~d}\left(N_{s}-\lambda s\right), \quad t \geq 0 .
$$

(1.2) However, the Brownian motion $\left(B_{t} ; t \geq 0\right)$ and the (standard) Gamma process $\left(\gamma_{t} ; t \geq 0\right)$, i.e., the subordinator with gamma laws

$$
\mathbb{P}\left[\gamma_{t} \in \mathrm{d} x\right]=\frac{x^{t-1} e^{-x} \mathrm{~d} x}{\Gamma(t)},
$$

share in fact more common properties. One of these common properties is quasi-invariance: for suitable functions $h$, the law of the process $B_{t}+\int_{0}^{t} h(s) \mathrm{d} s$ (resp. $\left.\int_{0}^{t} h(s) \mathrm{d} \gamma_{s}\right)$ is equivalent to the law of $B$ (resp. $\gamma$ ); on the quasi-invariance of Gamma, see Tsilevich-Vershik [15], Tsilevich-Vershik-Yor [16], and VershikYor [17].

The properties we shall be interested in concern the bridges associated to these processes. Properties relative to $B$ (resp. $\gamma$ ) will be denoted by $\left(1_{\mathrm{B}}\right)$, $\left(2_{\mathrm{B}}\right)$, etc. (resp. $\left(1_{\gamma}\right),\left(2_{\gamma}\right)$, etc.). The natural filtration of $B$ (resp. $\gamma$ ) will be called $\mathcal{B}$ (resp. $\mathcal{G}$ ).

The filtration of Brownian bridges is defined for $t \geq 0$ by

$$
\mathcal{B}_{t}^{*}=\sigma\left\{\frac{B_{u}}{u}-\frac{B_{v}}{v} ; u, v \in(0, t]\right\}=\sigma\left\{B_{u}-\frac{u}{t} B_{t} ; u \in[0, t]\right\} ;
$$

and the filtration of Gamma bridges by

$$
\mathcal{G}_{t}^{*}=\sigma\left\{\frac{\gamma_{u}}{\gamma_{v}} ; u, v \in(0, t]\right\}=\sigma\left\{\frac{\gamma_{u}}{\gamma_{t}} ; u \in[0, t]\right\} .
$$

These names, 'filtrations of bridges', are justified by property (3) below.

Quite fundamental is the independence property:

$\left(1_{\mathrm{B}}\right) \quad$ for every $t \geq 0, \mathcal{B}_{t}^{*}$ is independent of $\mathcal{B}_{t}^{+} \equiv \sigma\left\{B_{u} ; u \in[t, \infty)\right\}$;

$\left(1_{\gamma}\right) \quad$ for every $t \geq 0, \mathcal{G}_{t}^{*}$ is independent of $\mathcal{G}_{t}^{+} \equiv \sigma\left\{\gamma_{u} ; u \in[t, \infty)\right\}$.

In particular, $B_{t}\left(\right.$ resp. $\left.\gamma_{t}\right)$ is independent from $\mathcal{B}_{t}^{*}\left(\operatorname{resp} . \mathcal{G}_{t}^{*}\right)$, and for $t>0$ the inclusions $\mathcal{B}_{t}^{*} \subset \mathcal{B}_{t}$ and $\mathcal{G}_{t}^{*} \subset \mathcal{G}_{t}$ are strict. But this no longer holds in the large time limit:

$$
\begin{aligned}
& \mathcal{B}_{\infty}^{*}=\mathcal{B}_{\infty} ; \\
& \mathcal{G}_{\infty}^{*}=\mathcal{G}_{\infty} .
\end{aligned}
$$


From the independence property (1), it follows immediately that one may realize both the Brownian and Gamma bridges, on the time-interval $[0, t]$, starting at 0 , ending at $a$, as:

$$
\begin{gathered}
\left(B_{u}-\frac{u}{t} B_{t}+\frac{u}{t} a ; u \in[0, t]\right) ; \\
\left(a \frac{\gamma_{u}}{\gamma_{t}} ; u \in[0, t]\right) .
\end{gathered}
$$

The harness property was introduced by J. Hammersley [7], and has been discussed in particular by D. Williams [18]. In our opinion, it deserves to be better known; for a number of results and references, including some papers of P. Lévy, see Exercise 6.19 in Chaumont-Yor [4]. Examples of harnesses are all Lévy processes $X$ such that $\mathbb{E}\left[\left|X_{t}\right|\right]<\infty$, and all subordinators, whether integrable or not. A process $\left(X_{t} ; t \geq 0\right)$ is called a harness if, for all intervals $[a, b]$ and $[c, d]$ such that $[c, d] \subset[a, b]$, one has

$$
\mathbb{E}\left[\frac{X_{d}-X_{c}}{d-c} \mid \mathcal{X}_{a, b}\right]=\frac{X_{b}-X_{a}}{b-a},
$$

where $\mathcal{X}_{a, b}=\sigma\left\{X_{u} ; u \in[0, a] \cup[b, \infty)\right\}$. The observation that every integrable Lévy process verifies (4) is due to J. Jacod and P. Protter [9] (they do not use the term 'harness'); they furthermore deduce from (4) a property due to T. Kurtz: each integrable Lévy process $X$ satisfies, on the interval $[0, T]$,

$$
X_{u}=M_{u}^{(T)}+\int_{0}^{u} \frac{X_{T}-X_{s}}{T-s} \mathrm{~d} s,
$$

where $\left(M_{u}^{(T)} ; u \in[0, T]\right)$ is a martingale for the filtration $\mathcal{X}_{u}^{(T)}=\sigma\left\{X_{s}\right.$; $s \in[0, u] \cup\{T\}\}$.

In the case when the Lévy process is a Brownian motion or a Gamma process, the harness property can be understood as a consequence of a stronger property (where we still assume $[c, d] \subset[a, b]$ ):

$$
\begin{aligned}
& \frac{B_{d}-B_{c}}{d-c}-\frac{B_{b}-B_{a}}{b-a} \text { is independent from } \mathcal{B}_{a, b} ; \\
& \frac{\gamma_{d}-\gamma_{c}}{\gamma_{b}-\gamma_{a}} \text { is independent from } \mathcal{G}_{a, b} .
\end{aligned}
$$

(1.3) It has been remarked in Jeulin-Yor [10] that

$\left(7_{\mathrm{B}}\right) \quad$ the filtration $\left\{\mathcal{B}_{t}^{*}\right\}$ is generated by the Brownian motion

$$
B_{t}^{*}=B_{t}-\int_{0}^{t} \frac{\mathrm{d} u}{u} B_{u} .
$$


The corresponding property for the Gamma process is one of the main results of the present article: the filtration $\left\{\mathcal{G}_{t}^{*}\right\}$ is generated by the Gamma process

$$
\gamma_{t}^{*}=\sum_{s \in(0, t]} u_{s}\left(\frac{\Delta \gamma_{s}}{\gamma_{s-}}\right),
$$

where $u_{s}(x)$ is the function implicitly defined, for $x \geq 0$ and $s>0$, by

$$
\int_{u_{s}(x)}^{\infty} \frac{e^{-z}}{z} \mathrm{~d} z=\int_{x}^{\infty} \frac{\mathrm{d} y}{y(1+y)^{s}} .
$$

Finally, there is the property of time inversion for both Brownian motion and the Gamma process:

$\left(8_{\mathrm{B}}\right)$ the process $\left(t B_{\frac{1}{t}} ; t>0\right)$ is a Brownian motion;

$\left(8_{\gamma}\right)$ the process $\left(\ln \gamma_{\frac{1}{t}} ; t>0\right)$, or, for that matter, $\left(\ln \left(t \gamma_{\frac{1}{t}}\right) ; t>0\right)$, has independent, although non homogeneous, increments.

(1.4) The rest of this article is organized as follows:

- Section 2 gives references or proofs for properties (1) to (8).

- Section 3 investigates some properties of the Dirichlet processes

$$
D_{t}^{(T)}=\frac{\gamma_{t}}{\gamma_{T}}, \quad t \in[0, T],
$$

and argues in particular that from the observation of these processes it is possible to infer some properties of the Gamma process, thus going back and forth between the Gamma and Dirichlet processes.

- Section 4 raises related questions, among which the following: For which subordinators $\left(S_{t} ; t \geq 0\right)$ is the filtration

$$
\mathcal{S}_{t}^{*}=\sigma\left\{\frac{S_{u}}{S_{t}} ; u \in[0, t]\right\}
$$

equal to $\mathcal{S}_{t}$ ? different from $\mathcal{S}_{t}$ ?

If $S$ is a stable subordinator, it was shown in [14] that $\mathcal{S}^{*}=\mathcal{S}$; this will be proved again in Proposition 6. 


\section{§2. Properties (1) to (8)}

For Brownian motion, properties $\left(1_{\mathrm{B}}\right)$ to $\left(8_{\mathrm{B}}\right)$ are known. Property $\left(8_{\mathrm{B}}\right)$ is obtained by a simple correlation computation; $\left(1_{\mathrm{B}}\right)$ to $\left(7_{\mathrm{B}}\right)$ belong to the theory of Brownian bridges; for a general discussion of this topic, see chap. 1 of [19]. Concerning the harness property, besides the references given in the introduction, an unpublished manuscript by D. Williams conversely shows that, essentially, every continuous harness is a Brownian motion.

Property $\left(6_{\gamma}\right)$ is a particular instance of a more general statement: (9) the process

$$
\left(\frac{\gamma_{a+u}-\gamma_{a}}{\gamma_{b}-\gamma_{a}} ; u \in[0, b-a]\right)
$$

is a Dirichlet process $D^{(b-a)}$, with parameter $b-a$, independent of $\mathcal{G}_{a, b}$.

This has been known for a long time; for a general approach, with a measure space replacing the time-axis, see Ferguson [6]. The proof is quite elementary: it suffices to verify that, for $0=t_{0}<t_{1}<\cdots<t_{n}$, the vector

$$
\left(\frac{\gamma_{t_{1}}-\gamma_{t_{0}}}{\gamma_{t_{n}}}, \ldots, \frac{\gamma_{t_{n}}-\gamma_{t_{n-1}}}{\gamma_{t_{n}}}\right)
$$

is independent from $\gamma_{t_{n}}$, with density

$$
\frac{\Gamma\left(t_{n}\right)}{\prod_{i=1}^{n} \Gamma\left(t_{i}-t_{i-1}\right)} \prod_{i=1}^{n} x_{i}^{t_{i}-t_{i-1}-1}
$$

with respect to the Lebesgue measure $\mathrm{d} x_{1} \ldots \mathrm{d} x_{n-1}$ (or, as well, $\mathrm{d} x_{2} \ldots \mathrm{d} x_{n}$ ) on the simplex

$$
\left\{\left(x_{1}, \ldots, x_{n}\right): x_{i} \geq 0, x_{1}+\cdots+x_{n}=1\right\} ;
$$

and this is directly derived from the joint density of $\left(\gamma_{t_{1}}, \ldots, \gamma_{t_{n}}\right)$. A (much less elementary) converse is also known: Lukacs has proved in [13] that given two independent, strictly positive, nondegenerate random variables $X$ and $Y$, if $X+Y$ and $X /(X+Y)$ are independent, then $(X, Y) \stackrel{\text { law }}{=}\left(k \gamma_{u}, k\left(\gamma_{u+v}-\gamma_{u}\right)\right)$ for three parameters $k, u$ and $v$.

A large amount of literature is devoted to Dirichlet-type distributions, especially in view of Bayesian statistics; see for instance Diaconis-Kemperman [5].

As explained in the introduction, $\left(4_{\gamma}\right)$ and $\left(5_{\gamma}\right)$ stem from $\left(6_{\gamma}\right)$. Also, $\left(1_{\gamma}\right)$ is obtained from (9) by specializing $a=0$, and $\left(3_{\gamma}\right)$ follows from $\left(1_{\gamma}\right)$.

To establish $\left(2_{\gamma}\right)$, it suffices to observe that $\gamma_{n} / n \rightarrow 1$ a.s. when $n \rightarrow \infty$ by the law of large numbers; hence $\gamma_{t}=\lim _{n \rightarrow \infty}\left(n \gamma_{t} / \gamma_{n}\right)$ is $\mathcal{G}_{\infty}^{*}$-measurable. 
The rest of this section consists in establishing properties $\left(7_{\gamma}\right)$ and $\left(8_{\gamma}\right)$. Both are easy consequences of the next proposition.

Proposition 1. The point process

$$
\left\{\left(s, \frac{\Delta \gamma_{s}}{\gamma_{s-}}\right) ; s>0\right\}
$$

describing the "normalized" jumps of the Gamma process is an inhomogeneous Poisson point process, with intensity $\mathrm{d} s \frac{\mathrm{d} y}{y(1+y)}$, which generates the filtration $\left\{\mathcal{G}_{s}^{*} ; s>0\right\}$.

Equivalently, the point process

$$
\left\{\left(s, \frac{\Delta \gamma_{s}}{\gamma_{s}}\right) ; s>0\right\}
$$

is an inhomogeneous Poisson point process, with intensity $\mathrm{d} s \frac{\mathrm{d} u}{u(1-u)^{1-s}}$, generating $\mathcal{G}^{*}$.

Proof. Recall that the Lévy measure of the Gamma process is $\nu(\mathrm{d} x)=$ $\frac{e^{-x}}{x} \mathrm{~d} x$; and its Lévy exponent is $\ln (1+\lambda)$, because $\mathbb{E}\left[e^{-\lambda \gamma_{s}}\right]=\frac{1}{(1+\lambda)^{s}}=$ $\exp (-s \ln (1+\lambda))$.

Since the process $J_{s}=\Delta \gamma_{s} / \gamma_{s-}$ is $\mathcal{G}^{*}$-optional, to show the first claim it suffices to verify that, for every $\mathcal{G}^{*}$-predictable $H \geq 0$ and every Borel $f \geq 0$ null at 0 , one has

$$
\mathbb{E}\left[\sum_{s>0} H_{s} f\left(J_{s}\right)\right]=\iint \mathrm{d} s \frac{\mathrm{d} y}{y(1+y)^{s}} \mathbb{E}\left[H_{s}\right] f(y) .
$$

The left-hand side LHS (11) has the form $\mathbb{E}\left[\sum_{s} K\left(s, \omega, \Delta \gamma_{s}\right)\right]$, where

$$
K(s, \omega, x)=H_{s}(\omega) f\left(\frac{x}{\gamma_{s-}(\omega)}\right)
$$

is $\mathcal{G}$-predictable in $(s, \omega)$ and null for $x=0$. So LHS (11) can be computed with the Lévy system of $\gamma$, and one has

$$
\operatorname{LHS}(11)=\int \mathrm{d} s \int \nu(\mathrm{d} x) \mathbb{E}[K(s, \omega, x)]=\int \mathrm{d} s \int \nu(\mathrm{d} x) \mathbb{E}\left[H_{s} f\left(\frac{x}{\gamma_{s-}}\right)\right] .
$$


Inside the right-hand side expectation, we may replace $\gamma_{s-}$ by $\gamma_{s}$ (for each $s$, they are a.s. equal). Using now the independence of $H_{s}$ and $\gamma_{s}$ (because $H_{s}$ is $\mathcal{G}_{s}^{*}$-measurable and $\left(1_{\gamma}\right)$ holds), the expectation becomes $\mathbb{E}\left[H_{s}\right] \mathbb{E}\left[f\left(x / \gamma_{s}\right)\right]$; and to obtain (11), it suffices to write

$$
\begin{aligned}
\int \nu(\mathrm{d} x) \mathbb{E}\left[f\left(\frac{x}{\gamma_{s}}\right)\right] & =\mathbb{E}\left[\int_{0}^{\infty} f\left(\frac{x}{\gamma_{s}}\right) \frac{e^{-x}}{x} \mathrm{~d} x\right]=\mathbb{E}\left[\int_{0}^{\infty} f(y) \frac{e^{-\gamma_{s} y}}{y} \mathrm{~d} y\right] \\
& =\int_{0}^{\infty} f(y) \frac{\mathbb{E}\left[e^{-y \gamma_{s}}\right]}{y} \mathrm{~d} y=\int_{0}^{\infty} f(y) \frac{\mathrm{d} y}{y(1+y)^{s}} .
\end{aligned}
$$

The filtration $\mathcal{G}^{*}$ is generated by this Poisson point process because, for $0<s \leq t$,

$$
\frac{\gamma_{t}}{\gamma_{s}}=\prod_{r \in(s, t]} \frac{\gamma_{r}}{\gamma_{r-}}=\prod_{r \in(s, t]}\left(1+\frac{\Delta \gamma_{r}}{\gamma_{r-}}\right) .
$$

The last statement of Proposition 1 stems from the identity

$$
\frac{\Delta \gamma_{s}}{\gamma_{s}}=\frac{1}{1+1 /\left(\frac{\Delta \gamma_{s}}{\gamma_{s-}}\right)}
$$

and from the change of variable $u=\frac{1}{1+1 / y}$ in the intensity of $\frac{\Delta \gamma_{s}}{\gamma_{s-}}$.

Proposition 2 (property $\left(7_{\gamma}\right)$ ). For each $s>0$, the formula

$$
\int_{u_{s}(x)}^{\infty} \frac{e^{-z}}{z} \mathrm{~d} z=\int_{x}^{\infty} \frac{\mathrm{d} y}{y(1+y)^{s}} \leq+\infty
$$

defines a bijection $u_{s}: \mathbb{R}_{+} \rightarrow \mathbb{R}_{+}$. The sum

$$
\gamma_{t}^{*}=\sum_{s \in(0, t]} u_{s}\left(\frac{\Delta \gamma_{s}}{\gamma_{s-}}\right)
$$

is a.s. convergent and defines a Gamma process $\gamma^{*}$, which generates the filtration $\mathcal{G}^{*}$.

Proof. In view of Proposition 1, the sum (13) is convergent and defines a Gamma process if and only if $(s, x) \mapsto u_{s}(x)$ is measurable and, for almost all $s$, the image by $u_{s}$ of the measure

$$
\rho_{s}(\mathrm{~d} y)=\frac{\mathrm{d} y}{y(1+y)^{s}}
$$


is equal to the Lévy measure $\nu$ of the Gamma process. Remark that both measures $\rho_{s}$ and $\nu$ are infinite on the half-line $(0, \infty)$, but finite on every interval $[\varepsilon, \infty)$, and have strictly positive density on $(0, \infty)$; consequently, the function $u_{s}$ defined by (12), that is, by

$$
\nu\left(\left[u_{s}(x), \infty\right)\right)=\rho_{s}([x, \infty)),
$$

is an increasing bijection of $\mathbb{R}_{+}$onto itself, satisfying the above conditions. So $\gamma^{*}$ is a Gamma process for the filtration $\mathcal{G}^{*}$.

To see that $\gamma^{*}$ generates the filtration $\mathcal{G}^{*}$, call $v_{s}$ the reciprocal $\left(u_{s}\right)^{-1}$ of the bijection $u_{s} ; v_{s}$ is characterized by $\rho_{s}\left(\left[v_{s}(x), \infty\right)\right)=\nu([x, \infty))$. As $\Delta \gamma_{s} / \gamma_{s-}=v_{s}\left(\Delta \gamma_{s}^{*}\right)$ is adapted to the filtration of $\gamma^{*}$ and generates $\mathcal{G}^{*}$ (by Proposition 1), $\gamma^{*}$ generates $\mathcal{G}^{*}$.

Remark 1. Formula (13) giving $\gamma_{t}^{*}$ from $\gamma$ is not very pleasant: it cannot be transformed into a stochastic integral $\int_{0}^{t} H_{s} \mathrm{~d} \gamma_{s}$, with a $\mathcal{G}$-predictable $H$, because $u_{t}(x)$ is non linear in $x$. Does there exist another Gamma process $\widehat{\gamma}$, also generating $\mathcal{G}^{*}$, and such that $\widehat{\gamma}_{t}$ is a stochastic integral of some predictable process with respect to $\gamma$ ?

The answer is negative: in some sense, $\gamma^{*}$ is the simplest among all Gamma processes generating $\mathcal{G}^{*}$. More rigorously, if $\widehat{\gamma}$ is a Gamma process generating $\mathcal{G}^{*}$ and if, for every jump time $t$ of $\gamma$ (which is also a jump time of $\widehat{\gamma}$ ), the correspondence between $\Delta \gamma_{t}$ and $\Delta \widehat{\gamma}_{t}$ is monotone, then $\widehat{\gamma}=\gamma^{*}$.

To see this, observe that $\widehat{\gamma}$ and $\gamma^{*}$ are two Gamma processes generating the same filtration $\mathcal{G}^{*}$. Consequently (see Jacod [8], page 411), there exists a process $b(t, \omega, x), \mathcal{G}^{*}$-predictable in $(t, \omega)$, null for $x=0$, such that for almost all $(t, \omega)$, the map $x \mapsto b(t, \omega, x)$ is a $\nu$-preserving bijection of $\mathbb{R}_{+}$onto itself, that verifies

$$
\Delta \widehat{\gamma}_{t}(\omega)=b\left(t, \omega, \Delta \gamma_{t}^{*}(\omega)\right)
$$

If furthermore $\Delta \widehat{\gamma}_{t}$ is a monotone function of $\Delta \gamma_{t}$, it is also a monotone function of $\Delta \gamma_{t}^{*}$ (because $x \mapsto u_{t}\left(x / \gamma_{t-}\right)$ is strictly increasing), so, for almost all $(t, \omega)$, the bijection $x \mapsto b(t, \omega, x)$ is monotone. But there is only one monotone, $\nu$ preserving bijection, namely the identity map (because $\nu$ is infinite near 0 and finite at infinity). Hence $\Delta \widehat{\gamma}_{t}(\omega)=\Delta \gamma_{t}^{*}(\omega)$, and $\widehat{\gamma}=\gamma^{*}$.

Remark 2. Given $d \geq 1$ and any diffuse and unbounded Lévy measure $\ell$ on $\mathbb{R}^{d}$, there also exists a Lévy process $L$, with $\ell$ as Lévy measure and no Gaussian component, that generates the filtration $\mathcal{G}^{*}$. It suffices to construct $L$ such that $\Delta L_{t}=b\left(\Delta \gamma_{t}^{*}\right)$, where $b:(0, \infty) \rightarrow \mathbb{R}^{d} \backslash\{0\}$ satisfies $\nu \circ b^{-1}=\ell$. 
If furthermore $\ell$ is the Lévy measure of a subordinator, $L$ can be chosen as a subordinator, simply by setting $L_{t}=\sum_{s \in(0, t]} b\left(\Delta \gamma_{s}^{*}\right)$. Equivalently, this amounts to defining $L$ by

$$
L_{t}=\sum_{s \in(0, t]} w_{s}\left(\frac{\Delta \gamma_{s}}{\gamma_{s-}}\right)
$$

with $w_{s}$ such that $\rho_{s} \circ w_{s}^{-1}=\ell$, given for instance by $\ell\left(\left[w_{s}(x), \infty\right)\right)=$ $\rho_{s}([x, \infty))$, where $\rho_{s}$ is defined by $(14)$.

As for an example, if one wishes $L$ to be an $\alpha$-stable subordinator, with $0<\alpha<1$ and $\ell(\mathrm{d} z)=c z^{-1-\alpha} \mathrm{d} z$, it suffices to take

$$
w_{s}(x)=\left[\frac{\alpha}{c} \int_{x}^{\infty} \frac{\mathrm{d} y}{y(1+y)^{s}}\right]^{-\frac{1}{\alpha}} .
$$

Another example is the case of the measure $\rho_{s}$ itself. For each fixed $s>0$, $\rho_{s}$ is a Lévy measure; so there exists a subordinator $\left(Y_{t}^{(s)} ; t \geq 0\right)$ with this Lévy measure. It is possible to realize $Y^{(s)}$ in the filtration $\mathcal{G}^{*}$ by

$$
Y_{t}^{(s)}=\sum_{r \in(0, t]} v_{s}\left(\Delta \gamma_{r}^{*}\right)=\sum_{r \in(0, t]} v_{s} \circ u_{r}\left(\frac{\Delta \gamma_{r}}{\gamma_{r-}}\right),
$$

where $v_{s}$ (the reciprocal of $u_{s}$ introduced at the end of the proof of Proposition 2) transforms $\nu$ (the Lévy measure of $\gamma$ and $\gamma^{*}$ ) into $\rho_{s}$. This gives a "diagonal" expression of $\gamma^{*}$ :

$$
\gamma_{t}^{*}=\sum_{s \in(0, t]} u_{s}\left(\Delta Y_{s}^{(s)}\right),
$$

which is made possible by the joint choice of the family $\left(Y^{(s)} ; s>0\right)$ such that all the $Y^{(s)}$ have the same jump times.

Remark 3. Another class of processes generating $\mathcal{G}^{*}$ consists of the processes of the form

$$
R_{t}=\sum_{s \in(0, t]} h(s) g\left(\frac{\Delta \gamma_{s}}{\gamma_{s-}}\right),
$$

where $h>0$ on $(0, \infty)$, and $g$ is strictly increasing and null at 0 . As soon as $R$ is finitely valued, it has independent increments and generates $\mathcal{G}^{*}$ because of Proposition 1.

One can verify that

$$
\mathbb{E}\left[e^{-\lambda R_{t}}\right]=\exp \left[-\int_{0}^{t} \mathrm{~d} s \int_{0}^{\infty} \frac{1-e^{-\lambda h(s) g(y)}}{y(1+y)^{s}} \mathrm{~d} y\right],
$$


and derive therefrom that $R_{t}$ is a.s. finite if and only if

$$
\int_{0}^{t} \mathrm{~d} s \int_{0}^{\infty} \frac{1 \wedge h(s) g(y)}{y(1+y)^{s}} \mathrm{~d} y<\infty
$$

a sufficient condition is the finiteness of

$$
\mathbb{E}\left[R_{t}\right]=\int_{0}^{t} \mathrm{~d} s \int_{0}^{\infty} \frac{h(s) g(y)}{y(1+y)^{s}} \mathrm{~d} y .
$$

A particular case is $g(y)=y$, corresponding to

$$
R_{t}=\int_{0}^{t} h(s) \frac{\mathrm{d} \gamma_{s}}{\gamma_{s-}}
$$

in that case, one has the formula

$$
\mathbb{E}\left[R_{t}\right]=\int_{0}^{t} h(s) \mathbb{E}\left[\frac{1}{\gamma_{s}}\right] \mathrm{d} s=\int_{0}^{t} h(s)\left[\infty \mathbf{1}_{\{s \leq 1\}}+\frac{1}{s-1} \mathbf{1}_{\{s>1\}}\right] \mathrm{d} s,
$$

which is useless when $h>0$.

Another particular case is $g(y)=\ln (1+y)$, corresponding to

$$
R_{t}=\int_{0}^{t} h(s) \mathrm{d}\left(\ln \gamma_{s}\right)
$$

in that case, $\mathbb{E}\left[R_{t}\right]$ is more simply computed directly than via (15). One has $\mathbb{E}\left[R_{t}\right]=\int_{0}^{t} h(s) \mathrm{d} \mathbb{E}\left[\ln \gamma_{s}\right]$, and it suffices to compute $\mathbb{E}\left[\ln \gamma_{s}\right]$ : it is equal to

$$
\frac{1}{\Gamma(s)} \int_{0}^{\infty} x^{s-1} e^{-x} \ln x \mathrm{~d} x=\frac{1}{\Gamma(s)} \frac{\mathrm{d}}{\mathrm{d} s} \int_{0}^{\infty} x^{s-1} e^{-x} \mathrm{~d} x=\frac{\Gamma^{\prime}(s)}{\Gamma(s)}=\Psi(s),
$$

the logarithmic derivative of the gamma function; so

$$
\mathbb{E}\left[R_{t}\right]=\int_{0}^{t} h(s) \Psi^{\prime}(s) \mathrm{d} s .
$$

Taking the second derivative of $\ln \Gamma(s+1)=\ln s+\ln \Gamma(s)$ yields $\Psi^{\prime}(s+1)=$ $-\frac{1}{s^{2}}+\Psi^{\prime}(s)$. So $\Psi^{\prime}(s) \sim s^{-2}$ when $s \rightarrow 0$, and $\mathbb{E}\left[R_{t}\right]$ is finite if and only if $\int_{0}^{t} h(s) s^{-2} \mathrm{~d} s<\infty$.

Proposition 3 (property $\left(8_{\gamma}\right)$ ). The (increasing and left-continuous) process $\left(L_{t} ; t>0\right)$ defined by $L_{t}=-\ln \gamma_{\frac{1}{t}}$ has independent increments. More precisely, it has no continuous part, its jump process $\left\{\left(s, \Delta L_{s}\right) ; s>0\right\}$ is an inhomogeneous Poisson point process with intensity

$$
\frac{\mathrm{d} s}{s^{2}} \frac{e^{-\ell / s} \mathrm{~d} \ell}{\left(1-e^{-\ell}\right)},
$$


and its initial behavior at $t=0$ is given by

$$
L_{t}-\ln t=-\ln \left(t \gamma_{\frac{1}{t}}\right) \rightarrow 0 \quad \text { a.s. when } t \rightarrow 0 .
$$

Proof. It suffices to observe that, for $0<s<t$,

$$
L_{t}-L_{s}=\sum_{r \in\left(\frac{1}{t}, \frac{1}{s}\right]} \ln \left(1+\frac{\Delta \gamma_{r}}{\gamma_{r-}}\right)
$$

is measurable for $\mathcal{G}_{1 / s}^{*}$, and therefore independent of $\mathcal{G}_{1 / s}^{+}$by property $\left(1_{\gamma}\right)$. The structure of the jumps of $L$ is immediately obtained from the Poisson point process $\left\{\left(s, \frac{\Delta \gamma_{s}}{\gamma_{s-}}\right)\right\}$ with intensity $\mathrm{d} s \frac{\mathrm{d} y}{y(1+y)^{s}}$ (Proposition 1) by the change of variable $(s, y) \mapsto\left(\frac{1}{s}, \ln (1+y)\right)$. The initial condition means that $\frac{\gamma_{s}}{s} \rightarrow 1$ a.s. when $s \rightarrow \infty$; it comes from $\lim _{n \rightarrow \infty}\left(\frac{1}{n} \gamma_{n}\right)=1$ (law of large numbers) and from the trivial inequality

$$
\frac{\gamma_{n}}{n+1} \leq \frac{\gamma_{s}}{s} \leq \frac{\gamma_{n+1}}{n} \quad \text { for } n \leq s \leq n+1 .
$$

Remark 4. A similar change of variable shows that the filtration of the process $\left(\gamma_{\frac{1}{t}} ; t>0\right)$ (or, equivalently, of $L$ ) is also generated by the Gamma process

$$
\gamma_{t}^{\mathrm{inv}}=\sum_{s \geq t} f\left(s, \gamma_{\frac{1}{s}}-\gamma_{\frac{1}{s}-}\right)
$$

where $f$ is defined by

$$
\nu([f(s, x), \infty))=\frac{1}{s^{2}} \nu([x, \infty))
$$

and $\nu$ denotes the Lévy measure of $\gamma$.

\section{§3. Back and Forth between Gamma and Dirichlet Processes}

Fix a deterministic time $T>0$. Starting with the Gamma process $\gamma$ and its natural filtration $\mathcal{G}$, enlarge $\mathcal{G}$ by the knowledge of $\gamma_{T}$ at time zero:

$$
\mathcal{G}_{t}^{(T)}=\sigma\left\{\gamma_{s} ; s \in[0, t] \cup\{T\}\right\} .
$$


By property (9) with $a=0$ and $b=T$, on the interval $[0, T]$ the process $D_{t}^{(T)}=\gamma_{t} / \gamma_{T}$ is a Dirichlet process with parameter $T$ and law given by (10) with $T=t_{n}$; and $\mathcal{G}^{(T)}$ is the independent initial enlargement of its natural filtration $\mathcal{D}^{(T)}$ with the r.v. $\gamma_{T}$.

The Lévy system of the process $D^{(T)}$ on the interval $[0, T]$ can easily be derived from these properties. We denote by $\mathcal{P}\left(\mathcal{G}^{(T)}\right)$ the predictable $\sigma$-field for the filtration $\mathcal{G}^{(T)}$.

Proposition 4. Fix $T>0$. Let $H:[0, T] \times \Omega \times \mathbb{R}_{+} \rightarrow \mathbb{R}_{+}$be measurable for the product $\sigma$-field $\mathcal{P}\left(\mathcal{G}^{(T)}\right) \times \mathcal{B}\left(\mathbb{R}_{+}\right)$, and such that $H(t, \omega, 0) \equiv 0$. The $\mathcal{G}^{(T)}$-predictable compensator of the increasing process $\sum_{s \in(0, t]} H(s, \omega$, $\left.\Delta D_{s}^{(T)}(\omega)\right)$ is equal to

$$
\int_{0}^{t} \mathrm{~d} s \int_{0}^{1}(1-x)^{T-s-1} H\left(s, \omega,\left(1-D_{s-}^{(T)}\right) x\right) \frac{\mathrm{d} x}{x} .
$$

Proof. As $H$ may be multiplied by an arbitrary $\mathcal{G}^{(T)}$-predictable process, it suffices to check that both r.v.

$$
\sum_{t \in(0, T]} H\left(t, \omega, \Delta D_{t}^{(T)}(\omega)\right) \text { and } \int_{0}^{T} \mathrm{~d} t \int_{0}^{1}(1-x)^{T-t-1} H\left(t, \omega,\left(1-D_{t-}^{(T)}\right) x\right) \frac{\mathrm{d} x}{x}
$$

have the same expectation. Since, on the interval $[0, T]$, the filtration $\mathcal{G}^{(T)}$ is obtained from $\mathcal{D}^{(T)}$ by an independent enlargement (with $\gamma_{T}$ ), we may suppose that $H$ is $\mathcal{D}^{(T)}$-predictable. We may also restrict ourselves to the case when $H(t, \omega, x)=K(t, \omega) f(x)$, where both $K$ and $f$ are regular enough, $K$ vanishes on some interval $[T-\varepsilon, T]$ and $f$ on some interval $[0, \delta]$. For typographical simplicity we drop the superscript $(T)$. From the law of $D^{(T)}$, we know that, for $s<t$,

$$
\begin{gathered}
\mathbb{E}\left[f\left(D_{t}-D_{s}\right) \mid \mathcal{D}_{s}\right]=\frac{\Gamma(T-s)}{\Gamma(T-t) \Gamma(t-s)} \int_{0}^{1} f\left(\left(1-D_{s}\right) x\right)\left(D_{s} x\right)^{t-s}(1-x)^{T-t-1} \frac{\mathrm{d} x}{x} \\
=\frac{\Gamma(T-s)(t-s)}{\Gamma(T-t) \Gamma(1+t-s)} \int_{\delta}^{1} f\left(\left(1-D_{s-}\right) x\right)\left(D_{s-} x\right)^{t-s}(1-x)^{T-t-1} \frac{\mathrm{d} x}{x}
\end{gathered}
$$

Consider now the $n$-th dyadic subdivision $\left(t_{k}^{n}\right)$ of $[0, T-\varepsilon]$; notice that there are at most $1 / \delta$ values of $t$ (resp. $k$ ) such that $f\left(\Delta D_{t}\right) \neq 0\left(\right.$ resp. $\left.f\left(D_{t_{k+1}^{n}}-D_{t_{k}^{n}}\right) \neq 0\right)$. 
This implies

$$
\begin{aligned}
\mathbb{E}\left[\sum_{t} K_{t} f\left(\Delta D_{t}\right)\right] & =\mathbb{E}\left[\lim _{n} \sum_{k} K_{t_{k}^{n}} f\left(D_{t_{k+1}^{n}}-D_{t_{k}^{n}}\right)\right] \\
& =\lim _{n} \mathbb{E}\left[\sum_{k} K_{t_{k}^{n}} f\left(D_{t_{k+1}^{n}}-D_{t_{k}^{n}}\right)\right] .
\end{aligned}
$$

We may replace $f\left(D_{t_{k+1}^{n}}-D_{t_{k}^{n}}\right)$ by its conditional expectation on $\mathcal{D}_{t_{k}^{n}}$, namely

$$
\begin{aligned}
& \frac{\Gamma\left(T-t_{k}^{n}\right)\left(t_{k+1}^{n}-t_{k}^{n}\right)}{\Gamma\left(T-t_{k+1}^{n}\right) \Gamma\left(1+t_{k+1}^{n}-t_{k}^{n}\right)} \\
& \times \int_{\delta}^{1} f\left(\left(1-D_{t_{k}^{n}-}\right) x\right)\left(D_{t_{k}^{n}-} x\right)^{t_{k+1}^{n}-t_{k}^{n}}(1-x)^{T-t_{k+1}^{n}-1} \frac{\mathrm{d} x}{x} .
\end{aligned}
$$

The ratio $\Gamma\left(T-t_{k}^{n}\right) / \Gamma\left(T-t_{k+1}^{n}\right) \Gamma\left(1+t_{k+1}^{n}-t_{k}^{n}\right)$ is bounded above by the constant

$$
\frac{\Gamma(T) \vee \Gamma(\varepsilon)}{\inf _{v>0} \Gamma(v)^{2}}
$$

and tends to 1 , so in the limit, by dominated convergence,

$$
\mathbb{E}\left[\sum_{t} K_{t} f\left(\Delta D_{t}\right)\right]=\mathbb{E}\left[\int_{0}^{T} \int_{0}^{1} K_{t} f\left(\left(1-D_{t-}\right) x\right)(1-x)^{T-t-1} \frac{\mathrm{d} x}{x} \mathrm{~d} t\right] .
$$

Observe that letting $t$ tend to $s$ in (16) gives the infinitesimal generator of $D^{(T)}$ : for $f$ regular enough and null near 0 ,

$$
\left.\frac{\mathrm{d}}{\mathrm{d} t}\right|_{t=s} \mathbb{E}\left[f\left(D_{t}^{(T)}-D_{s}^{(T)}\right) \mid \mathcal{D}_{s}\right]=\int_{0}^{1} f\left(\left(1-D_{s-}^{(T)}\right) x\right)(1-x)^{T-s-1} \frac{\mathrm{d} x}{x} .
$$

This vanishing assumption is easily removed; but instead of repeating twice the same argument, it is more natural to directly derive the generator from Proposition 4, in the same spirit as in the next corollary.

Corollary 1. For any function $f$ on $\mathbb{R}_{+}$, with bounded variation on compacts, the process

$$
f\left(\gamma_{t}\right)-\int_{0}^{t} \mathrm{~d} s \int_{0}^{1} \frac{\mathrm{d} x}{x}(1-x)^{T-s-1}\left[f\left(\gamma_{s}+\left(\gamma_{T}-\gamma_{s}\right) x\right)-f\left(\gamma_{s}\right)\right]
$$

is a $\mathcal{G}^{(T)}$-local martingale on the interval $[0, T]$. 
Proof. We may suppose $f$ to be increasing. Writing

$$
f\left(\gamma_{t}\right)-f(0)=\sum_{s \in(0, t]}\left(f\left(\gamma_{s}\right)-f\left(\gamma_{s-}\right)\right)=\sum_{s \in(0, t]}\left(f\left(\gamma_{s-}+\Delta \gamma_{s}\right)-f\left(\gamma_{s-}\right)\right),
$$

it suffices to apply Proposition 4 to $H(s, \omega, x)=f\left(\gamma_{s_{-}}+x\right)-f\left(\gamma_{s_{-}}\right)$and to notice that $\gamma_{s-}=\gamma_{s}$ for all but countably many $s \in(0, t]$.

For instance, taking $f(y)=y$ gives $\left(5_{\gamma}\right)$ :

$$
\gamma_{t}-\int_{0}^{t} \frac{\gamma_{T}-\gamma_{s}}{T-s} \mathrm{~d} s \quad \text { is a } \mathcal{G}^{(T)} \text {-martingale on }[0, T] .
$$

More generally, T. Kurtz has obtained this formula for any integrable Lévy process; see Jacod and Protter [9].

As another example of application of Proposition 4, one also sees that, on the interval $[0, T)$, the increasing process

$$
\int_{0}^{t} \frac{\mathrm{d} D_{s}^{(T)}}{1-D_{s-}^{(T)}}=\sum_{s \in(0, t]} \frac{\Delta D_{s}^{(T)}}{1-D_{s-}^{(T)}}
$$

has independent increments; more precisely, its jumps $\frac{\Delta D_{s}^{(T)}}{1-D_{s-}^{(T)}}$ form a Poisson process on $[0, T) \times(0,1]$, with intensity $\mathrm{d} s(1-x)^{T-s-1} \mathrm{~d} x / x$.

Recall the notation introduced at the end of the proof of Proposition 2: for $s>0, v_{s}$ denotes the bijection $u_{s}^{-1}$ from $\mathbb{R}_{+}$to itself.

Proposition 5. Fix $T>0$. On the interval $[0, T]$ the process

$$
\mathbf{D}_{t}^{(T)}=1-\prod_{s \in(0, t]} \frac{1}{1+v_{T-s}\left(\Delta \gamma_{s}\right)}
$$

is a Dirichlet process with parameter $T$, generating on $[0, T]$ the same filtration as $\gamma$. More precisely, $\gamma$ can be recovered from $\mathbf{D}^{(T)}$ via the formula

$$
\gamma_{t}=\sum_{s \in(0, t]} u_{T-s}\left(\frac{\Delta \mathbf{D}_{s}^{(T)}}{1-\mathbf{D}_{s}^{(T)}}\right)
$$

Proof. By Proposition 2, we know that $\gamma_{t}^{*}=\sum_{s \in(0, t]} u_{s}\left(\frac{\Delta \gamma_{s}}{\gamma_{s-}}\right)$ is a Gamma process. As

$$
1+v_{s}\left(\Delta \gamma_{s}^{*}\right)=1+\frac{\Delta \gamma_{s}}{\gamma_{s-}}=\frac{\gamma_{s}}{\gamma_{s-}}
$$


one has

$$
D_{t}^{(T)}=\frac{\gamma_{t}}{\gamma_{T}}=\prod_{s \in(t, T]} \frac{\gamma_{s-}}{\gamma_{s}}=\prod_{s \in(t, T]}\left(1+v_{s}\left(\Delta \gamma_{s}^{*}\right)\right)^{-1} .
$$

By isomorphism, the process

$$
\bar{D}_{t}^{(T)}=\prod_{s \in(t, T]}\left(1+v_{s}\left(\Delta \gamma_{s}\right)\right)^{-1}
$$

is also a Dirichlet process with parameter $T$, on the interval $[0, T]$; notice that it depends on the jumps of $\gamma$ after $t$. Now reverse time on $[0, T]$ :

$$
\overleftarrow{\gamma}_{t}^{(T)}=\sum_{s \in[T-t, T]} \Delta \gamma_{s}=\gamma_{T}-\gamma_{(T-t)-}
$$

is another Gamma process on $[0, T]$, such that $\Delta \overleftarrow{\gamma}_{t}^{(T)}=\Delta \gamma_{T-t}$. And

$$
\overleftarrow{D}_{t}^{(T)}=\sum_{s \in[T-t, T]} \Delta \bar{D}_{s}^{(T)}=1-\bar{D}_{(T-t)-}^{(T)}
$$

is also a Dirichlet process on $[0, T]$, expressed in terms of the jumps of $\overleftarrow{\gamma}^{(T)}$ before $t$ :

$$
\begin{aligned}
\overleftarrow{D}_{t}^{(T)} & =1-\prod_{s \in[T-t, T]}\left(1+v_{s}\left(\Delta \gamma_{s}\right)\right)^{-1} \\
& =1-\prod_{s \in[0, t]}\left(1+v_{T-s}\left(\Delta \gamma_{T-s}\right)\right)^{-1}=1-\prod_{s \in(0, t]}\left(1+v_{T-s}\left(\Delta \overleftarrow{\gamma}_{s}^{(T)}\right)\right)^{-1}
\end{aligned}
$$

By isomorphism again, the process

$$
\mathbf{D}_{t}^{(T)}=1-\prod_{s \in(0, t]}\left(1+v_{T-s}\left(\Delta \gamma_{s}\right)\right)^{-1}
$$

is also a Dirichlet process with parameter $T$, clearly adapted to the filtration $\mathcal{G}$ generated by $\gamma$.

It remains to see that $\mathbf{D}^{(T)}$ generates $\mathcal{G}$. Call $Q_{s}$ the random variable $\left(1+v_{T-s}\left(\Delta \gamma_{s}\right)\right)^{-1}$, and write

$$
\Delta \mathbf{D}_{t}^{(T)}=\prod_{s \in[0, t)} Q_{s}-\prod_{s \in[0, t]} Q_{s}=\left(1-Q_{t}\right) \prod_{s \in[0, t)} Q_{s}=\left(1-Q_{t}\right)\left(1-\mathbf{D}_{t-}^{(T)}\right):
$$

equivalently,

$$
\frac{\Delta \mathbf{D}_{t}^{(T)}}{1-\mathbf{D}_{t-}^{(T)}}=1-\frac{1}{1+v_{T-t}\left(\Delta \gamma_{t}\right)}
$$


(By isomorphism, this gives another proof of the independence of the increments of (17).) When solving this for $v_{T-t}\left(\Delta \gamma_{t}\right)$, the left-limit $\mathbf{D}_{t-}^{(T)}$ disappears and there only remains

$$
v_{T-t}\left(\Delta \gamma_{t}\right)=\frac{\Delta \mathbf{D}_{t}^{(T)}}{1-\mathbf{D}_{t}^{(T)}},
$$

or

$$
\Delta \gamma_{t}=u_{T-t}\left(\frac{\Delta \mathbf{D}_{t}^{(T)}}{1-\mathbf{D}_{t}^{(T)}}\right)
$$

and finally

$$
\gamma_{t}=\sum_{s \in(0, t]} u_{T-s}\left(\frac{\Delta \mathbf{D}_{s}^{(T)}}{1-\mathbf{D}_{s}^{(T)}}\right) \quad \text { on }[0, T] .
$$

\section{$\S 4 . \quad$ Related Questions}

(4.1) A general time-reversal property? In the preceding proof, a timereversal of the interval $[0, T]$ plays a central rôle; on the other hand, in [10], formula $\left(7_{\mathrm{B}}\right)$ yielding the Brownian motion $B^{*}$ is established by letting a timereversal of $[0, T]$ act on the bridge associated to $B$. Are these two particular instances of a more general pattern? Is there something similar for other Lévy processes?

(4.2) Can a subordinator be recovered from its ratios? Let $\left(S_{t} ; t \geq 0\right)$ be some subordinator, with natural filtration $\mathcal{S}$. In view of the preceding study, it is natural to ask when the filtration

$$
\mathcal{S}_{t}^{*}=\sigma\left\{\frac{S_{u}}{S_{t}} ; u \in[0, t]\right\}
$$

is strictly included in $\mathcal{S}_{t}$. Notice that $\mathcal{S}^{*}$ is no longer independent from $S_{t}$, for, according to Lukacs [13], this independence essentially characterizes Gamma subordinators.

The next proposition shows that $\mathcal{S}^{*}=\mathcal{S}$ when $S$ is close enough to a stable subordinator.

Proposition 6. Let $S$ be a subordinator whose Lévy exponent $\psi(\lambda)$ verifies

$$
\psi(\lambda) \sim c \lambda^{\alpha} \quad \text { when } \lambda \rightarrow \infty
$$

for two constants $c>0$ and $\alpha>0$ (necessarily, $\alpha \leq 1$ ).

The filtrations $\mathcal{S}$ and $\mathcal{S}^{*}$ associated to $S$ are equal. 
This applies for instance to a sum of independent stable subordinators, or to the sum of a stable subordinator and an independent Gamma process.

Proof. It suffices to show that $S$ is adapted to $\mathcal{S}^{*}$; so, fixing $t>0$, we shall establish that $S_{t}$ is $\mathcal{S}_{t}^{*}$-measurable. To this end, consider the random variables

$$
X_{n}^{i}=\frac{1}{n^{\frac{1}{\alpha}}} \frac{1}{S_{\frac{i}{n} t}-S_{\frac{i-1}{n} t}} .
$$

For fixed $n$, the sequence $\left(X_{n}^{1}, \ldots, X_{n}^{n}\right)$ is i.i.d., with moments

$$
\begin{aligned}
M_{n}^{(1)}=\mathbb{E}\left[X_{n}^{i}\right] & =\mathbb{E}\left[\int_{0}^{\infty} e^{-\lambda / X_{n}^{i}} \mathrm{~d} \lambda\right] \\
& =\int_{0}^{\infty} \mathbb{E}\left[e^{-\lambda n^{\frac{1}{\alpha}} S_{\frac{t}{n}}}\right] \mathrm{d} \lambda=\int_{0}^{\infty} \exp \left[-\frac{t}{n} \psi\left(n^{\frac{1}{\alpha}} \lambda\right)\right] \mathrm{d} \lambda \\
M_{n}^{(2)}=\mathbb{E}\left[\left(X_{n}^{i}\right)^{2}\right] & =\mathbb{E}\left[\int_{0}^{\infty} e^{-\lambda / X_{n}^{i}} \lambda \mathrm{d} \lambda\right]=\int_{0}^{\infty} \exp \left[-\frac{t}{n} \psi\left(n^{\frac{1}{\alpha}} \lambda\right)\right] \lambda \mathrm{d} \lambda .
\end{aligned}
$$

Now the hypothesis on $\psi$ entails

$$
\lim _{n \rightarrow \infty} \frac{t}{n} \psi\left(n^{\frac{1}{\alpha}} \lambda\right)=t c \lambda^{\alpha}
$$

and also $\psi(\lambda) \geq \frac{c}{2} \lambda^{\alpha} \mathbf{1}_{\left[\lambda_{0}, \infty\right)}(\lambda)$ for some finite $\lambda_{0}$, whence, for all $n \geq 1$,

$$
\frac{t}{n} \psi\left(n^{\frac{1}{\alpha}} \lambda\right) \geq \frac{t}{n} \frac{c}{2} n \lambda^{\alpha} \mathbf{1}_{\left[n^{-\frac{1}{\alpha}} \lambda_{0}, \infty\right)}(\lambda) \geq t \frac{c}{2} \lambda^{\alpha} \mathbf{1}_{\left[\lambda_{0}, \infty\right)}(\lambda) .
$$

The estimate (19) gives an upper bound $M_{n}^{(2)} \leq a<\infty$ uniform in $n$; and, by dominated convergence, (18) and (19) imply that $M_{n}^{(1)} \rightarrow b=\int_{0}^{\infty} \exp \left(-c t \lambda^{\alpha}\right)$ $\mathrm{d} \lambda=(c t)^{-\frac{1}{\alpha}} \Gamma\left(\frac{1}{\alpha}+1\right)$ when $n$ tends to infinity.

Introduce the average

$$
Y_{n}=\frac{1}{n} \sum_{i=1}^{n} X_{n}^{i}
$$

One has $\mathbb{E}\left[Y_{n}\right]=\mathbb{E}\left[X_{n}^{i}\right]=M_{n}^{(1)} \rightarrow b$ and Var $Y_{n}=\frac{1}{n}$ Var $X_{n}^{i} \leq \frac{1}{n} M_{n}^{(2)} \leq$ $\frac{1}{n} a \rightarrow 0$; consequently, $Y_{n}$ converges in $\mathrm{L}^{2}$ to the constant $b$. And, since $b \neq 0$, $S_{t} Y_{n} / b$ converges in probability to $S_{t}$. But

$$
\frac{S_{t} Y_{n}}{b}=\frac{1}{b n^{\frac{1}{\alpha}+1}} \sum_{i=1}^{n} \frac{S_{t}}{S_{\frac{i}{n} t}-S_{\frac{i-1}{n} t}}
$$


is a function of the ratios $S_{\frac{i}{n} t} / S_{t}$, hence it is $\mathcal{S}_{t}^{*}$-measurable; and so is also its limit $S_{t}$.

(4.3) An ergodic transformation? Another question arising naturally from the present work is to study the transformation $\tau: \gamma \mapsto \gamma^{*}$ defined by $\left(7_{\gamma}\right)$; $\tau$ can be considered on the canonical path space associated to the Gamma process. More precisely, how does the loss of information expressed by the strict inclusion $\mathcal{S}_{t}^{*} \subsetneq \mathcal{S}_{t}$ carry over to the iterates $\tau^{n}$ of $\tau$ ? What do the corresponding $\sigma$-fields $\mathcal{S}_{t}^{* *}, \mathcal{S}_{t}^{* * *}, \ldots$ look like?

In [10], Jeulin and Yor establish that the transformation $B \mapsto B^{*}$ from $\left(7_{\mathrm{B}}\right)$ is ergodic (and, in fact, Bernoulli). Is there something similar for $\gamma$ ?

The non-linearity of $\tau$ seems to make difficult a direct approach to these questions.

(4.4) Explicit bridge formulae? Besides the Brownian motions and the Gamma processes, are there other Lévy processes $X$, with bridge $Y$ on $[0, T]$ given by some explicit affine formula of the form

$$
Y_{u}=a\left(u, T, X_{T}\right) X_{u}+b\left(u, T, X_{T}\right) ?
$$

(4.5) The Gamma process: a Krein functional of Brownian motion? As a (partial) consequence of the scaling property of Brownian motion, the stable subordinators can be realized from Brownian motion as $\int_{0}^{\tau_{t}} f\left(\left|B_{s}\right|\right) \mathrm{d} s$, where $\tau_{t}$ is the inverse Brownian local time, and $f$ a suitable power; more precisely, it is shown pp. 24-25 of [3] that, for any $\nu \in(0,1)$, one has:

$$
\mathbb{E}\left[\exp \left(-\frac{\lambda}{2} \int_{0}^{\tau_{t}} \mathrm{~d} s\left|B_{s}\right|^{\frac{1}{\nu}-2}\right)\right]=\exp \left(-t c_{\nu} \lambda^{\nu}\right),
$$

where

$$
c_{\nu}=\frac{\pi}{\nu \sin (\pi \nu)}\left(\frac{\nu^{\nu}}{\Gamma(\nu)}\right)^{2} .
$$

Using Kreı̆n's theory of strings, J. Bertoin ([1], [2]), F. Knight [11], and S. Kotani and S. Watanabe [12] have shown that many other subordinators admit similar representations. Is it possible to express the Gamma process as an additive functional of reflecting Brownian motion, considered at the inverse Brownian local time?

Professor S. Watanabe kindly informs us that he has shown that the Gamma process is the inverse local time at 0 of a reflecting gap diffusion on $[0, \infty)$ corresponding to some string (in the sense of M.G. Krel̆n); but it is not known if this string is strictly positive, so that the gap diffusion really is a diffusion. It would be very interesting to know how this string looks like! 


\section{Acknowledgement}

We are most grateful to Professors Shinzo Watanabe, Yoichiro Takahashi and Takashi Kumagai, and to the SEPO staff at RIMS, for their kind invitation and very friendly hospitality during the special year on Stochastic Analysis in 2002-2003.

\section{References}

[1] Bertoin, J., Subordinators: Examples and Applications, Lectures on Probability and Statistics, Ecole d'Eté de Probabilités de Saint-Flour XXVII-1997, Springer LNM, 1717 (1999).

[2] _ Applications de la théorie spectrale des cordes vibrantes aux fonctionnelles additives principales d'un mouvement brownien réfléchi, Ann. Inst. H. Poincaré Probab. Statist., 25 (1989), 307-323.

[3] Biane, P. and Yor, M., Valeurs principales associées aux temps locaux browniens, Bull. Sci. Math., 111 (1987), 23-101.

[4] Chaumont, L. and Yor, M., Exercises in Probability, Cambridge Univ. Press, 2003.

[5] Diaconis, P. and Kemperman, J., Some new tools for Dirichlet priors, Bayesian Statistics 5, J. M. Bernardo, J. O. Berger, A. P. Dawid and A. F. M. Smith eds., 97-106. Oxford University Press, 1996.

[6] Ferguson, T. S., A Bayesian analysis of some nonparametric problems, Ann. Stat., 1 (1973), 209-230.

[7] Hammersley, J. M., Harnesses, Proc. Fifth Berkeley Sympos. Mathematical Statistics and Probability, Vol. III: Physical Sciences., Univ. California Press, Berkeley, 89-117, 1967.

[8] Jacod, J., Calcul stochastique et problèmes de martingales, Springer LNM, 714 (1979).

[9] Jacod, J. and Protter, P., Time reversal on Lévy processes, Ann. Prob., 16 (1988), $620-641$.

[10] Jeulin, T. and Yor, M., Filtration des ponts browniens et équations différentielles linéaires, Séminaire de Probabilités XXIV, 227-265, Springer LNM, 1426 (1990).

[11] Knight, F.B., Characterization of the Lévy measures of inverse local times of gap diffusion, Seminar on Stochastic Processes, 1981., Prog. Prob. Statist., 1 53-78, Birkhäuser 1981.

[12] Kotani, S. and Watanabe, S., Kreĭn's spectral theory of strings and generalized diffusion processes, Functional analysis in Markov processes (Katata/Kyoto 1981), Springer LNM, 923 (1982), 235-259.

[13] Lukacs, E., A characterization of the gamma distribution, Ann. Math. Stat., 26 (1965), 319-324.

[14] Pitman, J. and Yor, M., Arcsine laws and interval partitions derived from a stable subordinator, Proc. London Math. Soc., 65 (1992), 326-356.

[15] Tsilevich, N. and Vershik, A., Quasi-invariance of the gamma process and multiplicative properties of the Poisson-Dirichlet measures, C. R. Acad. Sci. Paris Sér. I, 329 (1999), 163-168.

[16] Tsilevich, N., Vershik, A. and Yor, M., An infinite dimensional analogue of the Lebesgue measure and distinguished properties of the gamma process, J. Funct. Anal., 185 (2001), 274-296.

[17] Vershik, A. and Yor, M., Multiplicativité du processus gamma et étude asymptotique des lois stables d'indice $\alpha$, lorsque $\alpha$ tends vers 0. Prépubl. 289, Laboratoire de Probabilités, Paris, 1995. 
[18] Williams, D., Some basic theorems on harnesses, Stochastic Analysis (a tribute to the memory of Rollo Davidson), D. Kendall and H. Harding eds. Wiley, 1973.

[19] Yor, M., Some Aspects of Brownian Motion. Part I: Some Special Functionals, Lectures Math. ETH Zürich, Birkhäuser 1992. 